\title{
Variations
}

Variations

Revue internationale de théorie critique

$24 \mid 2021$

Echos

\section{Théorie critique, sujets politiques et la situation brésilienne}

Entretien avec Bruna Coelho, Marialia Mello Pisani, Felipe Ribeiro, Fernanda Azeredo

\section{Vladimir Safatle}

Traducteur : Daphné Anton

\section{(2) OpenEdition}

Journals

Édition électronique

URL : https://journals.openedition.org/variations/2005

DOI : 10.4000/variations.2005

ISSN : 1968-3960

Éditeur

Les amis de Variations

Référence électronique

Vladimir Safatle, "Théorie critique, sujets politiques et la situation brésilienne », Variations [En ligne], 24 | 2021, mis en ligne le 03 juillet 2021, consulté le 07 juillet 2021. URL : http://

journals.openedition.org/variations/2005; DOI : https://doi.org/10.4000/variations.2005

Ce document a été généré automatiquement le 7 juillet 2021.

Les ami•e•s de Variations 


\section{Théorie critique, sujets politiques et la situation brésilienne}

Entretien avec Bruna Coelho, Marialia Mello Pisani, Felipe Ribeiro, Fernanda Azeredo

Vladimir Safatle

Traduction : Daphné Anton

\section{NOTE DE L'ÉDITEUR}

Présentation du collectif : B. Coelho est doctorante en philosophie à Paris 8 , M. Mello Picani est professeure de philosophie à l'Université fédérale ABC (Sao Paolo), F. Ribeiro est professeur assistant au département de psychologie à l'Université du Para, F.

Azeredo est doctorante en anthropologie à l'Ehess.

1 Question : Une des idées centrales à partir de laquelle vous pensez les sujets politiques contemporains est celle de la détresse - Hilflosigkeit ${ }^{1}$ - que vous récupérez au sein d'une perspective freudienne - au-delà de la demande de soin. Ce concept peut apparaître, selon vos mots, comme « une espèce de précondition affective nécessaire à l'ouverture à l'inconnu, à l'impensé, à ce qui nous meut en tant que force hétéronome, qui est profondément privée de la place de l'autre $»^{2}$. Pour commencer, pouvez-vous simplement nous introduire à cette idée d'hétéronomie sans servitude que vous avez développée dernièrement?

Vladimir Safatle : Je me suis demandé qu'elle serait la base affective de l'émancipation sociale, car cette question interroge ce qui organise la structure des motivations de l'action émancipatrice. Je pense que cette question de l'émancipation n'est pas seulement liée à une idée d'autonomie de construction, d'autolégislation, d'autogouvernement et d'autogestion. Elle est aussi, et principalement, liée à la capacité d'ouverture aux causalités extérieures, hors de nos projections et de nos attentes, qui nous affectent. Autrement dit, il est question d'une certaine structure relationnelle et de l'affirmation de cette dynamique relationnelle comme 
caractérisation fondamentale de l'émancipation. L'émancipation est pensée généralement à partir de la forme de l'autonomie. Mais je trouve cette perspective un peu individualiste, une sorte de collectif qui a toujours été pour moi une espèce d'individu amplifié. J'ai trouvé qu'il serait plus adéquat de penser quelles seraient les bases affectives d'une structure relationnelle dans laquelle je suis capable de m'ouvrir à ce que je ne contrôle pas. Et c'est de là que vient la question de la détresse.

(tion: On peut reconnaitre dans cette notion d'hétéronomie sans servitude la présence de l'anti-idéalisme francfortien, selon lequel « le Bien en soi, le Bien absolu ne peut être énoncé mais seulement ses excès peuvent être décrits $»^{3}$. On comprend alors que la progression récente de vos recherches se soit dirigée vers l'analyse du sens de la dialectique chez Theodor Adorno. Pourriez-vous exposer les idées centrales de ce dernier livre, Dar corpo ao Impossivel ${ }^{4}$ qui essaie d'établir les connexions existantes entre cette œuvre et l'idée de l'hétéronomie sans servitude?

Vladimir Safatle : J'ai voulu faire ce travail sur Adorno car la dialectique négative me paraissait être un bon moyen de discuter comment les expériences de non-identité et de négativité sont formatrices d'une nouvelle figure du sujet. Et, vous voyez, penser cette idée de causalité extérieure fondamentale à la constitution d'une certaine émancipation, c'est aussi une façon de penser les relations structurantes de la nonidentité. Comme je viens d'un dialogue avec la psychanalyse, il est pour moi très clair que tout cela tourne un peu autour de ce que signifie un sujet affecté par son inconscient. Un inconscient qui n'est pas simplement dérivé d'une philosophie de l'autoréflexion, ni de l'élargissement de la capacité de compréhension de la conscience, mais qui est en fait une autre dimension de l'expérience, de la perception et de l'organisation du sens qui s'ouvre au sujet, ou qui l'affecte.

Question : Une autre dimension de la structure psychique donc?

6 Vladimir Safatle : Oui. J'insisterais particulièrement sur ce point. C'est un autre mode d'organisation de l'expérience qui affecte le sujet, qui n'est pas le mode d'organisation propre de la conscience. Cela dit, il m'a paru d'un côté que l'expérience intellectuelle adornienne était incompréhensible sans reconnaître l'importance de ce type de débat. Mais il y a plusieurs questions qui structurent l'écriture de ce livre. A l'horizon de la théorie critique actuelle, il m'a paru très important de revenir aux dimensions radicales de la première génération de l'École de Francfort. Je pense qu'il y a eu une domestication de la théorie critique à partir de sa reprise habermassienne qui a influencé la seconde et la troisième génération d'intellectuels, sans parler de la quatrième. Elle a consisté, entre autres choses, à abandonner le concept de révolution comme ce qui ordonne la critique politique et à retourner à une conception du sujet qui était en fait une réification de la figure de l'individu libéral. Une idée de la reconnaissance fondée sur une base anthropologique, dans laquelle la reconnaissance de l'individualité, de l'intégrité personnelle, finit par être l'horizon régulateur fondamental. Enfin, tout cela m'a paru être extrêmement douteux et d'une régression absolue.

7 Question : Peut-on alors dire que votre livre rompt avec ce destin de la théorie critique, celui des solutions pacificatrices qui réinvestissent les facultés transcendantales qui structuraient le sujet ou le vieux sujet autonome de la philosophie ?

8 Vladimir Safatle : Oui, bien qu'ils aient fait la critique de la philosophie de la conscience, ils n'ont pas vu qu'en travaillant à partir de cette perspective de 
l'autonomie, sous une forme proto-kantienne, ce problème réapparaitrait forcément d'une façon ou d'une autre.

Question : Vous réhabilitez alors un certain transcendantalisme à travers d'autres moyens, avec par exemple le caractère structurant du langage, même si on retrouve dans les théories de la reconnaissance un mouvement semblable.

Vladimir Safatle : Oui, mais je pense que cette théorie réhabilite la philosophie de la conscience de telle façon qu'ils ne l'auraient jamais accepté, et c'est pourtant ce qui est arrivé. Donc j'ai eu d'un côté pour projet de récupérer un peu de la radicalité de l'expérience francfortienne en insistant sur la nécessité de la dialectique comme figure fondamentale de la pensée critique, car ces figures post-habermassiennes ne sont pas dialectiques.

11 Question : Peut-on dire qu'il y a une tentative d'étouffer la dialectique, de s'éloigner intellectuellement des contradictions immanentes?

12 Vladimir Safatle : Oui, c'est pour cela que j'ai voulu d'un côté récupérer la dialectique dans sa dimension de théorie du mouvement à partir des contradictions immanentes. Et cela vaut autant pour l'organisation de l'expérience sociale que pour l'organisation de l'expérience psychique. De l'autre, il était question pour moi de récupérer une tradition de la pensée dialectique au Brésil que je pense être un élément fondamental de l'organisation de l'expérience nationale. Je pense que la grande pensée critique du Brésil est une pensée qui a pour matrice un type de dialectique qui s'est appuyé sur Adorno et sur la dialectique négative pour critiquer une certaine idée de la dialectique, encore d'actualité, liée à cette balance entre les opposés qui serait propre à la situation périphérique du pays. Une sorte de conciliation entre les contraires, une notion très pacifiée de la dialectique. Alors que cette pensée critique brésilienne a investi une dimension non réconciliée de la dialectique. Je voulais reprendre un peu ça. Je pense que, de fait, notre condition historique actuelle l'oblige. Enfin, il y a eu un troisième aspect de mon travail qui, depuis mon premier livre A Paixão do Negativo ${ }^{5}$ consistait à contribuer à l'organisation d'un débat entre la pensée française contemporaine et les traditions qui se reconnaissent d'une façon ou d'une autre dans la philosophie hégélienne, bien que de façon polémique et agonistique. C'est comme ça que j'ai fait mon premier livre sur Lacan, dans lequel j'ai insisté un peu sur cette articulation, et ce livre Dar corpo ao Impossível achève ce cycle.

13 Question : Il vient néanmoins un peu à contrecourant de certains mouvements de la pensée dialectique brésilienne et de beaucoup d'auteurs qui pensent la théorie critique en s'appuyant sur la seconde génération. Beaucoup soulignent cet héritage dans la pensée critique.

14 Vladimir Safatle : Je pense qu'ils font une lecture caricaturée de la philosophie française, qui est un peu celle du Discours philosophique de la modernité de Habermas. Ils sont des figures conservatrices du rationalisme.

15 Question : A propos du Discours philosophique de la modernité, comment vous répondez à un des principaux arguments de la théorie habermassienne concernant l'émancipation selon lequel toute critique sociale présuppose un horizon normatif, utopique ou rationnel, en tant que condition de réalisation, comme si sans idéal de normativité il n'était possible de fonder une critique?

16 Vladimir Safatle : Le fondement de la critique est donné par la souffrance. Elle impulse la critique. Cette expérience de la souffrance a de multiples enracinements. Toute la 
question passe, entre autres choses, par une compréhension de la nature de la souffrance comme déclencheur de la critique. Ce n'est pas seulement une souffrance liée à l'échec d'attentes normative et de justice. Je pense qu'il y a des dimensions fondamentales de la souffrance qui sont liées au fait que les processus de socialisation et d'individuation relatifs à l'action sociale sont en eux-mêmes contradictoires, c'est-àdire qu'ils fonctionnement bien quand ils fonctionnent mal. Autrement dit, ils sont incapables de s'opérer sans produire de la souffrance. C'est un peu une vision freudienne classique du processus civilisationnel: la souffrance, le sentiment de culpabilité, la névrose, etc., ne sont pas produit par une déviation de ce processus. Non. C'est son mouvement normal qui implique la production de tel type de souffrance. Et il y a alors deux options : ou bien naturaliser la souffrance, ou bien la comprendre comme le moteur fondamental de la critique qui vise des formes de vie encore non configurées, absentes de l'horizon de ce processus civilisationnel. Rassembler la psychanalyse et la théorie de la révolution, le marxisme, c'est une autre façon de créer un " freudomarxisme ", qui à mon avis est une manière beaucoup plus intéressante d'aborder la question que de dire : "Où est-la base normative de votre critique ? La critique ne peut se retourner contre cette normativité sans rencontrer le risque de créer des contradictions performatives ». Cela ne vaut pas grand-chose car ça ne prend pas en compte une autre tradition qui pense ces formes de vies qui non seulement ne réalise pas les valeurs et les normes en vigueur, mais qui comprennent que la seule façon de réaliser ces valeurs c'est précisément de les abandonner. C'est un peu l'idée d'Adorno quand, par exemple, il parle de la notion de progrès. Il n'en vient jamais à l'abandonner mais il insiste sur le fait que le progrès commence là où il se termine. C'est-à-dire que l'unique manière de réaliser l'élan initial de cette notion est d'en faire la critique radicale, c'est de l'abandonner. Bien sûr, pour quelqu'un qui renonce à la dialectique, cela n'a pas de sens de dire ces choses-là, pour cette personne la proposition est sémantiquement inconsistante. Mais de là vient l'importance de se comprendre afin de récupérer la dialectique comme élément fondamental de l'horizon fondamental de la critique.

Question : Peut-on dire que vous mobilisez une espèce de souffrance ontologique, de souffrance fondamentale? Les sujets traverseraient l'histoire non parce qu'ils sont mus par la volonté de justifier un certain horizon normatif, mais parce qu'ils souffrent ontologiquement? Pouvez-vous nous parler un peu d'où vous tenez cette notion de souffrance fondamentale?

Vladimir Safatle : Tout d'abord, je ne me réfère pas exactement à une souffrance ontologique, mais plutôt au fait que les formes de souffrance produites par les configurations de reproduction matérielle de la vie sont le moteur de la recherche de nouveauté, de détachement de ces dynamiques de reproduction matérielle. C'est à dire que les configurations historiques locales produisent et créent des expériences de souffrance qui sont des éléments fondamentaux pour toute critique. Je pense qu'il serait irréel de ne pas considérer cette expérience de configuration générale des actions sociales. Par exemple un de mes travaux a consisté à discuter la souffrance de détermination, en renversant cette idée au fond durkheimienne d'anomie. Il est possible de comprendre la souffrance de détermination comme un élément fondamental de la vie contemporaine, comme une limitation de certaines figures déterminées du "je », de la subjectivité, de la vie sociale et de l'identité. Même si la détermination est construite de façon différente selon les circonstances, je pense cependant que l'indétermination peut être comprise comme l'élément principal de la 
liberté. C'est pour cela que la souffrance de détermination est insupportable, parce qu'elle limite quelque chose de fondamental d'une expérience qui, bien qu'elle soit historiquement située (c'est une question difficile à avancer sans connaissances anthropologiques) est pour nous très évidente. Mais d'où vient ce concept de souffrance ? Il vient d'une articulation entre la psychanalyse et la dialectique. Ces figures de la souffrance, qui sont déjà apparues chez Hegel, sont évidentes : l'angoisse, le vide, par exemple. La figure classique de la souffrance chez Marx est l'aliénation. Elle est une forme de souffrance sociale fondamentale et il y a à cet égard une chose intéressante par rapport à Freud : l'aliénation n'est pas le résultat d'une dérégulation $\mathrm{du}$ fonctionnement de la vie sociale, mais elle est plutôt constitutive du fonctionnement normal de la société capitaliste.

Question : En ce sens, Marx se rapproche de Freud?

Vladimir Safatle : Oui, au moins sur ce point. Freud dit aussi que la souffrance dont traite la psychanalyse est le résultat du fonctionnement normal de la famille, du travail, des processus civilisationnels, de l'Etat, de l'Eglise, des forces armées, de la vie institutionnelle. Vous pouvez lire ces considérations sur la production de la souffrance en considérant que ce constat est notre horizon final. Mais vous pouvez également penser de la façon suivante : «S'il en est ainsi, nous sommes autorisés à penser un autre type de processus civilisationnel ». C'est comme ça que s'inscrit, à mon sens, la ligne de partage entre deux types d'interprétation de ces traditions.

Question : Pour en revenir à l'indétermination, à travers cette idée de la détressea ${ }^{a}$ qui apparaît dans des textes comme L'Avenir d'une Illusion ou Le Malaise dans la culture, Freud démontre comment celle-ci résulte de dynamiques psychiques basées sur des idéalisations, qui rendent la relation du sujet avec l'autre davantage marquée par la servitude et la dépendance que par l'émancipation ou la liberté. La détresse ne seraitelle pas la cause de positions sociales régressives?

Vladimir Safatle : En effet, il existe cette transformation de la détresse en une demande illusoire de protection. Mais il existe aussi une autre position qui est la position analytique. Dans l'Avenir d'une Illusion, Freud décrit la vision du monde scientifique comme pour l'homme l'aveu de la Kleinheit, de "toute sa détresse, de sa petitesse dans l'ensemble de l'univers ${ }^{6}$ et de la contingence. C'est ainsi que l'idée de détresse apparaît. Je pense donc que votre question est pertinente. Mais lorsqu'on considère que la détresse peut conduire à la fois à la régression et à une forme de courage nécessaire à l'émancipation, cela nous pousse à nous demander comment s'effectue ce passage et dans quelles conditions ces positions sociales régressives sontelles possibles?

Question : Venons-en aux analyses que vous faites de l'actualité au sein du débat public et à un type de radicalité semblable à celui de vos critiques et analyses philosophiques. Vos diagnostiques insistent sur le fait que, tout au long du développement du capitalisme, les modèles de conciliation ont fini par atteindre leurs limites. La radicalisation apparaitrait alors comme une alternative nécessaire. Plutôt que d'être tenté par une forme de régression psychique, il serait temps de ne plus craindre de penser et d'oser l'impensable. D'un autre côté, quand vous abordez des sujets plus techniques et spécifiques de la politique réelle, vous revendiquez un agenda de reformes. Par exemple, celles du système électoral participatif, du système fiscal, de la politique macroéconomique du gouvernement. Vous indiquez donc des chemins très concrets de changements institutionnels. De ce point de vue, ne resterait-il au sujet 
qu'une révolution sans grand récit, ni mégalomanies téléologiques, dans le but d'apporter des réformes concrètes et nécessaires ? Comment pourrait-on mettre ces deux positions sur le même plan, à savoir d'un côté le renoncement aux utopies et à l'énonciation de l'avenir, et de l'autre l'appel à prendre position en faveur de mesures politiques concrètes?

Vladimir Safatle : On m'a déjà signalé cette apparente contradiction. Un discours qui insiste sur une dynamique de radicalisation tout en évoquant certaines situations concrètes paraît réformiste. Je comprends le problème la manière suivante. Premièrement, je ne pense pas que la question fondamentale soit de savoir s'il fait sens ou non de faire des propositions à l'échelle locale d'une situation politique concrète. Le système électoral fonctionne de telle manière que lorsque vous gagnez une élection, vous faites, par exemple, une réforme fiscale. Puis vous faites la défense de l'impôt sur les grandes fortunes, de l'augmentation de l'impôt sur les héritages, de façon progressive, dans un pays où les riches ne sont taxés qu'à $7,5 \%$. Tout ça donne l'impression de ressembler à de la vieille politique sociale-démocrate. L'aspect du problème le plus important pour moi est celui de l'horizon politique. Admettre que, dans certaines situations, il est nécessaire de faire un pas après l'autre, ne signifie pas l'abandon de la perspective révolutionnaire Il y a là une opposition assez puérile, mais assez fréquente, entre s'engager à ne pas participer de l'intérieur à ces situations concrètes et faire tabula rasa, et ne gérer que les aspects limités du capitalisme actuel. Je pense qu'il n'est question ni de l'un ni de l'autre, car le problème me semble être à un autre niveau. Je crois que la révolution n'est pas quelque chose qui se prépare, voilà peut-être la différence. Elle arrive très souvent en dépit de ce que les sujets qui la font pensent être en train de faire. Nous connaissons beaucoup de processus révolutionnaires dans lesquels les sujets qui y participaient n'avaient pas conscience de cet aspect proprement révolutionnaire. Dans la Révolution française, la prise de conscience de la révolution fut un processus lent, qui prit forme peu à peu, comme si les personnes étaient prises dans une dynamique historique qu'ils ne pouvaient pas encore projeter. En ce sens, Laclau affirme que personne ne sort dans la rue en disant " Nous souhaitons la fin du capitalisme ! ", sinon quelques groupes très limités. Les demandes sont plutôt « Nous voulons la terre, le pain et la paix $»^{7}$ de telle sorte qu'un programme social-démocrate réformiste pourrait y répondre. Mais pourquoi n'est-ce pas le cas ? Non pas parce que ces demandes sont formulées par une avant-garde révolutionnaire, mais parce que leur véritable réalisation implique le dépassement de tout un système économique. Si nous admettons que la révolution est un événement et non un projet, alors nous comprenons deux choses : que lorsqu'elle se produit, il faut être prêt, et que, si elle ne se produit pas, il faut faire pression, forcer de l'intérieur ou de l'extérieur du gouvernement. Ce qui peut se passer - et c'est le plus grand de tous les risques - c'est qu'au début d'un processus de transformation sociale, les forces qui l'opèrent s'accommodent d'un horizon limité et se restreignent à le gérer de l'intérieur. C'est ainsi que les processus historiques capitulent, c'est-à-dire que les forces qui apparaissent dans le champ politique en tant que forces de transformation radicale commencent à opérer des transformations et une dynamique de changement ponctuels...

Question : Qui peuvent être cooptées par des forces conservatrice...

Vladimir Safatle : Tout à fait. Donc je ne vois pas de contradiction lorsque j'insiste sur un processus de radicalisation et que je considère, en même temps, certaines situations 
locales. Je dis que nous pouvons essayer et présenter un programme, défendre un agenda concret. Et il existe deux façons de le faire qui vont ensemble : la première est d'affirmer un horizon pour nos actions et la seconde est de le considérer seulement comme une première étape, puisque d'autres viendront - même s'il est évident qu'il existe aussi une dynamique du maintien de l'insatisfaction populaire et de retardement de la réalisation des transformations sociales.

Question : Comme vous, avec d'autres, le remarquez, les extrêmes droites qui montent aujourd'hui annoncent elles aussi une révolution, et ce faisant, dirigent les masses vers des transformations concrètes. Sont-elles également des révolutions?

Vladimir Safatle : Oui, et je dirais même pire. Les extrêmes droites ne font que reprendre et mettre en circulation ce terme. Voilà tout le problème. Elles ont récupéré la dynamique anti-institutionnelle. L'extrême droite sera d'autant plus forte le jour où elle sera la seule à manier ce vocabulaire. Et il n'y a qu'un seul moyen de rompre cette force, c'est de diviser ce désir anti-institutionnel. Il n'y a plus une alternative à gauche pour ce désir et d'ailleurs, l'expérience de la gauche des vingt dernières années a été une catastrophe. Remarquez une chose étrange : il y a cinq ans, nous étions au milieu de la crise grecque. Syrisa est apparu comme une grande promesse pour la gauche mondiale. Ils sont arrivés à une situation limite et ont organisé un référendum pour décider si la Grèce devait, en fin de compte, accepter ou non le fameux mémorandum de la Troïka, et en cas de refus, mettre au moins un pied en dehors de la communauté européenne. Que s'est-il passé ? Tout le monde s'en souvient bien : le peuple a dit non et le gouvernement a fait marche arrière.

Question : Et Aléxis Tsípras est allé discrètement à la BCE négocier la dette publique, le plan d'austérité...

Vladimir Safatle : Il a tué dans l'œuf toutes les forces et la mobilisation possibles d'un nouveau moment pour la gauche mondiale! Et cela n'a pas été qu'un problème grec. Parce que si la Grèce avait respecté le référendum, il y aurait eu une déferlante sur l'Europe. Et qu'est ce qui se passe cinq ans plus tard ? L'extrême droite récupère ce thème de la résistance à la communauté européenne, et ça donne le Brexit. D'une certaine façon, la droite prend le relai de la gauche, là où cette dernière a échoué. La gauche devient le gestionnaire du système actuel, avec un cœur soi-disant plus humain. A quoi ça sert tout ça ? Les secteurs les plus défavorisés, ceux qui ne s'identifie déjà plus à l'ordre actuel, suivent désormais l'extrême droite. Ils disent qu'elle tient ses promesses, alors que la gauche, lorsqu'elle arrive au pouvoir, se désiste de ses engagements. Voilà ce qui démontre impuissance et faiblesse aux yeux de la population. Pis, que la gauche n'a plus lieux d'être, qu'elle n'a plus de lieux, tout court, au sens fort du terme. La gauche est morte. Il serait important qu'elle comprenne ça, qu'elle commence par là. Elle est morte parce qu'elle n'a pas réussi, de manière globale, à être un vecteur de transformation effective de la société. Elle apporte des solutions ponctuelles, s'occupe des droits et répond mieux aux problèmes de la reconnaissance, ici ou là, mais elle n'a pas de projet de transformation globale.

31 Question : Mais il semble que l'extrême droite non plus n'a pas de tel projet. Elle ne fait que simuler une révolution puisqu'en vérité, elle suit l'agenda néolibéral.

32 Vladimir Safalte : Certes, c'est là toute la question. Elle propose un programme tellement radicalisé du néolibéralisme qu'elle le fait passer pour une rupture. C'est ce que font les néolibéraux, il n'y a qu'à lire les livres de Mises. Il prétend que le libéralisme n'est jamais arrivé, qu'il n'y a eu que de courtes périodes mises en échec par 
l'étatisme. Le néolibéralisme devient une utopie. Et la stratégie de l'extrême droite est précisément d'affirmer que le néolibéralisme n'a en fait jamais existé, qu'il n'a jamais vraiment été tenté. Au Brésil, l'argument que les extrêmes droites utilisent est de prétendre que la gauche est au pouvoir depuis la fin de la dictature militaire. C'est du délire! C'est une stratégie très claire. Elles se déresponsabilisent du résultat catastrophique qu'a produit la démocratie brésilienne et elles se présentent ensuite comme la vraie rupture, alors que leurs principes idéologiques se limitent à l'application radicale du modèle néolibéral. La gauche n'a pas réussi à affirmer de façon crédible : "Nous n'avons rien à voir avec ce modèle». Et quand elle est arrivée au pouvoir, elle en est devenue le gestionnaire, non sans quelque honte. Souvenez-vous de l'élection de Dilma Roussef en 2014. Elle a gagné avec 51,64\% des voix, c'était sûr que le pays allait se diviser. Et qu'est-ce que vous faites quand votre pays se divise? Vous organisez votre camp politique, parce qu'il va y avoir des tensions. Vous gouvernez d'abord pour les vôtres. Et c'est ce que fait maintenant Bolsonaro, qui gouverne d'abord pour son propre camp politique, et pour les autres, advienne que pourra. La gauche, quant à elle, fait appel aux sommités de la pensée conservatrice, du libéralisme économique, elle donne le ministère de l'économie à Joaquim Levy ${ }^{8}$, le ministère de l'agriculture à Katia Abreu'. Qu'est-ce que la gauche montre en faisant ainsi? Elle montre que lorsque les problèmes commencent à arriver, il n'y a que les néolibéraux qui sont capables de les résoudre, que ce sont eux qui font les choses sérieusement.

Question: Vous soutenez donc que la gauche devrait se radicaliser, qu'elle devrait antagoniser son discours?

Vladimir Safatle : Oui, en effet. Car en agissant comme le gouvernement l'a fait, il a ainsi affirmé l'impossibilité d'une politique effective de gauche, qui ne serait viable qu'en temps de prospérité, et que ce sont les néolibéraux qui ont une réponse concrète à la crise. La gauche a perdu l'occasion de radicaliser son discours, ce que fait l'extrême droite.

Question: Que pensez-vous des disputes identitaires dans le camp de la gauche actuelle? Certains prétendent que ces questions sont secondaires et superflues, des "écrans de fumées" qui détournent l'attention de la gauche des prétendus vrais problèmes économiques.

Vladimir Safatle : Ce n'est certainement pas mon avis. Au contraire, je pense que ce que l'on a l'habitude aujourd'hui d'appeler des questions «identitaires » sont des questions centrales. Je ne ferai jamais une telle distinction entre les questions économiques et celles à propos de la reconnaissance, puisqu'elles sont liées entre elles et ce de façon évidente depuis le fascisme du début du XXème siècle. Par exemple, un des éléments fondamentaux de la mobilisation des fascistes a été le combat contre ce qu'ils appelaient le "bolchévisme sexuel », résultat des premières années de la révolution russe, avec la modification radicale de la structure familiale, de la position des femmes, des mouvements d'égalitarisme radical. A ce moment, l'URSS avait le code civil le plus avancé sur ces questions: la possibilité du divorce, le premier mariage homosexuel fut célébré dans les années 20 en Union Soviétique... Je pense donc que ces deux questions, identitaires et économiques, vont ensemble. Et l'extrême droite en a tellement conscience qu'elle ne peut abandonner aucun de ces thèmes, sinon elle laisse la porte ouverte à une forme de perception et de sensibilité sociale qui est radicalement contraire à la forme de vie sociale qu'elle cherche à imposer. Mais le point n'est pas là, car en fait ces problèmes ne sont pas identitaires. Je crois voir ici une stratégie 
néolibérale fondamentale qui consiste à éliminer la force transformatrice de ces luttes en les soumettant à une grammaire identitaire qui n'est pas la leur. Je dirais même que ces luttes sont les vraies luttes universalistes. Ce sont elles qui disent que l'universalité ne s'est pas encore réalisée puisqu'il existe encore ces chaines spécifiques de violences contre les Noirs, les femmes, les LGBT, qui doivent avant tout être brisées. Et pour que l'universalité se réalise, il faut d'abord nommer. Dire qu'en plus de la violence qui existe déjà dans la vie sociale, il existe, par exemple, la violence spécifique contre les Noirs, indépendamment de leur classe, de leur genre, de leur religion, et la nommer comme une violence motivée par la race. Surtout à l'intérieur d'un pays comme le Brésil qui a pour expérience fondamentale l'esclavagisme, où le latifundium esclavagiste est la base de la vie sociale jusqu'aujourd'hui. Le problème est que ces luttes sont prises entre deux tendances contradictoires. C'est vrai qu'il existe à l'intérieur de ces luttes des tendances identitaires qui les reformulent avec la grammaire libérale. Ces tendances luttent pour un espace propre, un espace "à soi ", sans pour autant remettre en cause la grammaire d'où émergerait cet espace. Pourtant, cette force identitaire, en émergeant, peut fracturer cette grammaire et faire éclater les luttes restreintes à "ce qui m'est propre ». Et il est vrai que le libéralisme n'a pas le moindre problème avec ces luttes, jusqu'à un certain point.

Question : On voit les publicités télévisées se dire pro-féministes et pro-identitaires...

Vladimir Safatle: Mais ceci n'est rien de plus qu'une stratégie néolibérale d'absorption de la force transformatrice de ces luttes. Car cette force dépasse cela et insiste sur le fait qu'un type d'expérience égalitaire où les relations de genre sont marquées par l'oppression subsistent n'est plus possible. Souvenez-vous de ce que Marx disait déjà au $19^{\text {ème }}$ siècle : "Vous voulez comprendre le niveau de développement d'une société ? Regardez quelles relations elle entretient avec les femmes. Et là vous saurez où en est cette société. » Steve Bannon dit quelque chose en ce sens (les canailles ont cet avantage, ils finissent toujours par dire les choses explicitement) : «A chaque fois que la gauche parle des politiques d'identité, je gagne. $»^{10} \mathrm{Il}$ cherche à pousser la gauche à parler des identités. Puisqu'en fait le vrai problème n'est pas la question de l'identité ainsi formulée. Ce qui est en jeu, c'est la construction d'un vrai espace d'indifférenciation que n'existe pas encore.

Question : Etant donné que beaucoup de mouvements sociaux emploient une logique identitaire au moment d'organiser leurs luttes, ne faudrait-il pas discuter, au sein de la théorie critique, le recours à la désidentification, afin de rendre compte de ce problème de l'ontologie politique dans le processus historique? Autrement dit, penser en termes d'identité peut-il porter préjudice à la compréhension des rapports qui lient les luttes entre elles? Comment pourrions-nous penser les mouvements sociaux au-delà de l'identité, sans tomber dans un processus de désidentification abstraite?

Vladimir Safatle : Tout d'abord je n'ai jamais été contre les usages provisoires de l'identité. Ces usages existent d'ailleurs de fait, et ils sont stratégiquement nécessaires pour la raison suivante: ils donnent une visibilité au processus et aux systèmes de violence. Ces questions apparaissent donc quand ces usages cessent d'être provisoires. Et je pense que ce qui est caractéristique de la perte du caractère provisoire de ces usages, c'est l'effondrement de la solidarité générale, lorsqu'elle devient difficile à opérer. La solidarité générale, c'est dire ceci : «L'oppression que je subis m'ouvre aux autres formes d'oppression existantes, à tous les niveaux, de genre, de race, de classe, de religion.» Ce sont donc la population trans, les Palestiniens, les travailleurs blancs 
pauvres, ce sont ceux qui sont complètement détachés de toute forme d'identification avec le mode hégémonique de reproduction sociale, par conséquent, la classe de ceux qui sont déconnectés est aussi l'ensemble de ceux qui n'admettent pas être des reproducteurs de ce processus. Il y a comme une espèce de transversalité qui peut se produire, et se reproduisant, modifier tous les éléments qui sont en relation, parce qu'ils consolident tous, d'une certaine manière, un type de circulation et de transmigration. Il est donc clair que l'enjeu pour la droite est d'empêcher qu'une telle chose arrive. Car quand elle arrive, quand ces rapports étranges et monstrueux s'installent, alors les résistances aux formes de vie hégémoniques émergent de tous les côtés. La lutte se transforme complètement, elle cesse d'être sectorisée, elle se pulvérise. Il y a là une véritable dynamique de construction hégémonique. La dynamique de la solidarité ne résulte donc pas d'une association de demandes particulières car elle brise la particularité des demandes.

Question : Ernest Laclau construit son concept de "signifiant vide " à partir de la discussion post-marxiste selon laquelle il y a des chaînes d'équivalences qui se forment à l'intérieur d'une société libérale démocratique représentative, il y a la possibilité que se forment de grandes hégémonies populistes qui feront face au pouvoir. Vous rapprochez-vous de la théorie de Laclau?

Vladimir Safatle : Je ne pense pas qu'il y ait une chaîne d'équivalence, mais plutôt un flux de mutations. C'est la différence. Chaîne d'équivalence signifie qu'il n'existe plus de figure politique unifiée, comme le prolétariat, qui est alors fragmenté, et que les demandes multiples doivent rencontrer un point d'équivalence. Ce point d'équivalence est donné, entre autres choses, par la relation de tous à une espèce de "signifiant vide ", qui va servir un peu de point de capiton [Lacan], de processus de capitonnage. Qu'arrive-t-il à l'intérieur de cette dynamique ? Outre le point d'équivalence, apparaît le problème de l'exclusion mutuelle. C'est ce que Laclau ne voit pas et qu'il ne parvient pas à penser. Il ne voit pas le populisme comme une stratégie de paralysie de la société, mais il pense, en dernière instance, qu'il existe des chaînes que créeront de nouveaux processus dynamiques qui pourrait construire "le peuple » en tant qu'hégémonie, mais il oublie complètement que le populisme est aussi un mode de gestion de la paralysie sociale. Les processus populistes ont la caractéristique de ne pas aller jusqu'au bout, arrivés à un certain point, ils s'arrêtent. Pourquoi? Parce que les demandes commencent à s'annuler. Regardez ce qu'a été le gouvernement de Perón en Argentique. Ce gouvernement était en même temps composé de trotskistes et de représentants de l'oligarchie agraire argentine : c'était sûr qu'ils finiraient par mettre le pied sur le frein. On ne pense pas ces paralysies continues. Pour moi, l'expérience brésilienne en est la meilleure expression. D'abord, il y a une gestion claire de la paralysie [le gouvernement Lula], ensuite, que se passe-t-il ? Précisément ce à quoi nous sommes en train d'assister : l'extrême droite arrive au pouvoir et nous dit que la société était paralysée, et que pour y remédier, elle doit rompre et cesser de négocier. Elle veut diviser la société, et c'est ce qu'elle fait. Au-delà de ça, il serait intéressant de que nous pensions d'autres formes de construction de cette espèce de subjectivité générale, non abstraite. Je trouve ça drôle quand j'entends «Ah! mais ça c'est une conception complètement abstraite ! », car il n'y a rien d'abstrait là-dedans. Pourquoi y aurait-il quelque chose d'abstrait dans le fait de penser que les gens ne luttent pas simplement pour leurs prédicats? dans le fait de penser qu'ils ne luttent pas simplement pour ce qui leur appartiendraient en propre? Ils luttent également pour quelque chose qui les dépasse, ils luttent pour un espace qui réponde à leur désir de ne 
pas être déterminé et visibilisé, à leur désir d'imperception, d'anonymat, de transformation. Alors, pourquoi cela est vu comme une abstraction, tandis que la défense des attributs propres est vu comme une chose concrète ? Cela n'est rien d'autre qu'un dogme métaphysique selon lequel la prédication est un élément fondamental de la détermination de l'existence. Les personnes qui adoptent ce discours sont aristotéliciennes sans le savoir.

Question : Nous voudrions aborder maintenant un thème qui n'est pas directement présent dans vos travaux, mais qui nous paraît fondamental dans le contexte politique international actuel, à savoir la question écologique, et notamment à propos de l'environnement et de l'Amazonie. Herbert Marcuse nous invite à penser cette question à partir d'une théorie critique dont l'horizon est révolutionnaire. Nous pensons notamment à trois textes, "Ecologie et Révolution " ", "Ecologie et la critique de la société moderne $»^{12}$, "Nature et Révolution $»^{13}$. Il semble que nous pourrions rapprocher de ces textes certaines de vos affirmations à propos du rôle de la subjectivité et des affects à l'intérieur d'une théorie critique et d'une pratique révolutionnaire. La question écologique n'est cependant pas une question centrale dans vos ouvrages, bien que vous vous inspiriez de certains thèmes centraux de l'œuvre de Marcuse, notamment dans votre dernier livre, Dar corpo ao Impossivel. Cette année, avec l'augmentation des incendies en Amazonie et avec la violence perpétrée contre les peuples amazoniens, nous voyons circuler dans le discours international le thème de la protection de la nature. Comment voyez-vous l'émergence de ce débat dans le discours international?

Vladimir Safatle : Ce rapprochement est très important. C'est un thème que j'aimerais développer davantage. Il y a diverses manières d'approcher le problème. Peut-être, un des aspects fondamentaux de la théorie de l'émancipation serait de comprendre qu'il n'y a pas d'émancipation des sujets sans une émancipation des choses. Qu'est-ce que cela signifie? Qu'il faut cesser d'appréhender les choses comme les expressions d'un sujet, comme propriété potentielle, comme ce qui est nécessairement assujetti, à l'intérieur de la division classique personne/chose, en tant que cette dernière est "soumise à ». C'est une tendance de la théorie de l'émancipation, de donner de l'autonomie aux personnes en soumettant les choses. Cela vaut également pour la relation à la nature. Mais il existe, dans la nature, une modification structurelle où elle cesse d'être vue comme un espace potentiel de propriété, que ce soit d'un peuple, d'un individu ou de quoi que ce soit d'autre, où elle accède à un autre statut. En ce qui concerne le Brésil, cela me parait un élément absolument central, pour deux raisons. Premièrement, la logique coloniale à laquelle nous avons assisté cette année est tout simplement la continuation de la logique coloniale du Brésil depuis 1500. Prenez par exemple Mario Pedrosa, le plus grand critique d'art que ce pays ait eu. Je prends son exemple seulement pour indiquer jusqu'où va ce raisonnement, jusqu'où il est assumé. Mario Pedrosa fait une distinction entre le modernisme mexicain et le modernisme brésilien, et dit en gros que le modernisme mexicain a dû entrer en relation avec une culture autochtone, précolombienne, fortement présente là-bas. Cela produit une chose hybride, comme l'a été le muralisme $\mathrm{e}^{14}$ par exemple. Le modernisme brésilien n'avait pas cette forte culture autochtone avec laquelle il aurait été nécessaire se lier, et il s'est donc senti plus libre dans la reconstruction de l'espace au sein l'architecture moderne. Je me souviens avoir lu cela et d'avoir été frappé par la violence d'un tel constat. Et Pedrosa n'a même pas dû s'en rendre compte ni s'être exclamé: "Comment ça il n'existe pas de culture autochtone au Brésil ? ». C'est ça l'illusion coloniale au Brésil : il 
n'y avait rien avant, ce n'était que de la brousse qu'il fallait incendier et passer au bulldozer, parce que le développement devait permettre de prendre possession du territoire. C'est l'idée à la base de la construction de Brasilia, car quand Lucio Costa ${ }^{15}$ s'expliquera sur la forme en avion ou en croix de la capitale, il dira que cela correspond à «l'acte de prendre possession ». Quel type de pays est celui qui construit son rapport à soi sous la forme d'une dépossession, afin d'en permettre l'appropriation? La question intéressante est: pourquoi cette grammaire-là ? Pourquoi ne pas rendre compte d'une expérience dans laquelle il est question de la présence d'une multiplicité de peuples sur un territoire irréductible à une quelconque appropriation? Ce sont des espaces que je ne peux m'approprier, car ce sont eux qui me désapproprient. Je dirais qu'une des expériences esthétiques fondamentales du pays fût celle qui tenta de donner forme à cela. Prenez la littérature de Guimarães Rosa, qui est, d'une certaine manière, une puissance politique qui nous dit que nos rêves [de développement] ont produit des monstres et que le pire d'entre eux est celui qui fait du sertã $0^{16}$ un espace nul, où il n'y a rien. Ecoutez pourtant ce qu'il y a: des couches et des couches d'expériences qui s'imbriquent les unes dans les autres et font résonner toute l'expérience du monde.

Question : Ce qui est présent chez lui dans toute la langue vivante, n'est-ce pas ? C'est un aspect intéressant.

Vladimir Safatle : Tout à fait, il crée un langage aux multiples temporalités. Le jagunço [ndt : homme de main, criminel] de Guimarães parle de cette façon : « en lui, il y avait quelque chose de pyrrhonien ${ }^{17}$. Mais quel jagunço fait ça ? D'où vient-il ? Où est-il ? Ce que Guimarães voulait dire, c'est que la forme délirante de développement qui a été mise en place a été d'une violence brutale. Le problème est que ce développementalisme fou a sa forme militarisée, que représente Bolsonaro. Ce dernier se demande bien pourquoi on se chamaille autour d'un buisson, puisqu'en fin de compte, le pays a été construit en rasant et en détruisant tout. Et il s'offusque en disant: «Pourquoi toute cette confusion maintenant? Juste parce que le président français a fait un scandale ?!». Après les incendies de l'Amazonie, sa popularité a augmenté et n'a pas diminué ! Il est étonnant de voir à quel point nous ne nous sommes pas arrêtés un instant pour penser ce que cela voulait dire. Dans la vie nationale, vous avez une conscience populaire qui considère la nature est l'ennemi qu'il faut apprivoiser, nous approprier. Donc, j'insiste, cet élément est l'un des plus ancrés dans l'expérience nationale et il mérite une critique, un travail plus profond. Je parlais de Mario Pedrosa, mais c'est un travail qui ne passe pas seulement par la droite. Vous auriez vu quel était le plan de développement écologique de la gauche au pouvoir... rappelez-vous simplement les déclarations effrayantes d'Aldo Rebelo ${ }^{18}$, qui avait quelque chose d'un développementalisme stalinien tout à fait primaire. C'était ça le gouvernement de la gauche.

47 Question : Quelles sont les impasses écologiques des politiques de développement de l'Etat appliquées au Brésil ces dernières années, que ce soit par le gouvernement actuel ou par les précédents ? Quelles sont les impasses en face desquelles la question écologique met les gouvernements qui se disent de gauche ? Quelle idée du communisme pourrait nous aider à traiter la question écologique, la protection de la nature, des communautés locales et des connaissances traditionnelles?

48 Vladimir Safatle: C'est une question intéressante. La différence entre la politique écologique mise en œuvre précédemment et celle de Bolsonaro est une différence quantitative, et non qualitative. C'est l'intensité de l'impact qui est différent. Le PT au 
gouvernement a fait Belo Monte ${ }^{19}$, et Lula a dit « si ces petits poissons sont le problème, nous les mettrons dans le palais du Planalto ». L'impact était évidemment différent, mais pas la logique de ce que signifie le problème écologique du Brésil.

Question : Quand Lula est arrivé au pouvoir, il a élu une administration de militaires en Amazonie.

Vladimir Safatle : Tout à fait. Nous sommes en train de parler d'un pays qui a connu deux catastrophes écologiques en un $a^{20} . \mathrm{Il}$ y a eu le problème du pétrole dans les plaines du Nordeste, deux catastrophes écologiques dans l'état du Minais Gerais dues à un développement prédateur et destructif. La dernière rupture du barrage dans le Minais a fait 200 morts. Aucun acteur politique pertinent n'a réussi à placer l'agenda écologique au centre des préoccupations. Au fond, il s'agit tout simplement de la notion classique du colonialisme, le pillage. Rien de plus. Et ceci dans un pays où le parti écologiste est inexistant.

Question: Pensez-vous à un programme écologique, une façon de concilier développement et préservation de l'environnement?

Vladimir Safatle : Je pense que ce serait un des axes fondamentaux pour ressusciter la gauche, car la gauche brésilienne n'existe plus. Il faut déjà commencer par-là, son cycle s'est terminé, car elle est désormais incapable de réagir à la hauteur des évènements, elle ne fait que délirer sur certains sujets. Par exemple, elle continue, jusqu'à aujourd'hui, à se disputer sur ce qu'il s'est passé en 2013, s'il s'agissait ou non d'un complot de la CIA. En Angleterre par exemple, si le parti travailliste perd une élection, il crée un shadow cabinet, un cabinet fantôme, pour continuer de surveiller et de s'opposer au gouvernement. Tandis qu'ici, la gauche n'arrive pas à faire quelque chose de semblable. Nous ignorons ce qu'elle veut vraiment, quel est son programme économique, ce qu'elle compte faire. Va-t-elle revenir sur la réforme des retraites si, par exemple, le gouverneur de Bahia (membre du PT) la met en place dans son État? Nous ne savons pas quelle réforme politique la gauche brésilienne propose, quelle transformation elle préconise, ce qu'elle veut mettre à la place de ce que nous avons aujourd'hui. Va-t-elle revenir à l'expérience de coalition de 2013, pour la énième fois ? Personne ne le sait et elle non plus. Cela signifie simplement une chose : la gauche est morte. Et pour ressusciter, pour se réinventer, il va falloir qu'elle le reconnaisse. L'un des thèmes classiques de cette mort est justement l'absence brutale, dans un pays comme le Brésil, d'un débat structuré sur le problème écologique, c'est-à-dire un débat réel et concret. Vous demandez "comment concilier développement et écologie ?", mais le débat doit d'abord commencer par une interrogation sur la nature même de ce développement, sur ce que l'on entend réellement par là. Il s'agit d'une série de présupposés anthropologiques les plus évidents. Parce qu'on part du principe que ce qui serait, disons, le mode de vie des populations indigènes au Brésil ne s'est pas suffisamment développé. Mais développé par rapport à quoi, à qui ? Qui donne le critère ? La Banque mondiale internationale ? Le FMI ?

Question : Dans l'opinion publique, le développement c'est la soi-disant « reprise de la croissance ", l'amélioration de la balance commerciale, le reprise de l'industrialisation du Brésil...

Vladimir Safatle: Le développement est entouré d'une série de clichés que les journalistes diffusent. J'entends parler de cette « reprise de la croissance » depuis que je suis enfant. Je n'ai jamais réussi à comprendre réellement ni ce que veut dire « reprise », ni ce qu'est « croissance» dans ce contexte. Des phrases comme «le bond 
de la balance commerciale » se banalise d'une manière impressionnante. Tout ceci est très abstrait. Qu'est-ce que cela représente réellement dans la vie concrète des gens ? Vous voyez, la pauvreté est un fait relatif, en relation avec quelque chose. Marx dit que le sujet qui habite dans les Samoa de l'huile de poisson et de la pêche ne se sent pas pauvre. Il désire et transforme d'une autre manière. Autant dire qu'une telle idée a été supprimée de l'horizon national: la possibilité même de vivre dans une diversité de mode de développement. Et les libéraux du pays défendent l'idée que les masses de travailleurs ont une vie meilleure. Mais encore une fois, meilleure par rapport à quoi et à quelles circonstances?

Question: De façon générale, la gauche brésilienne s'insère dans une grammaire libérale. Le PT [Parti des Travailleurs], à travers récemment Fernando Haddad ${ }^{21}$, essaie de remettre la question des banques à l'ordre du jour, de donner la priorité au secteur productif du pays pour relancer l'emploi et augmenter le revenu national, afin que le Brésil se relève économiquement. Ciro Gomes ${ }^{22}$ fait également partie de ceux qui ont recours à ces thèmes, qui critique l'économie de rente et les banques. S'agit-il d'une gauche isolée?

Vladimir Safatle: Cette gauche fait partie d'un horizon populiste et développementaliste. Cette critique disparaît dès qu'ils entrent au gouvernement, au prétexte qu'on ne peut lutter contre un secteur fondamental de l'économie et qu'il faut donc faire des ajustements ponctuels. Le Brésil connaît bien cette logique, nous y avons déjà été confronté et il n'y pas de raisons cela ne se reproduise pas à nouveau. Je pense néanmoins que vous posez une question intéressante, car c'est de fait l'horizon dans lequel s'inscrit le débat des idées et, en dernière instance, de l'action. La gauche brésilienne reste cependant prise en otage par cet horizon depuis les années 40 et la Seconde Guerre Mondiale. C'est d'ailleurs le seul qu'elle connaisse. Pourtant, le résultat est toujours le même. J'ai récemment sorti un recueil de textes de Carlos Marighella. La critique qu'il formula à sa sortie du Parti communiste brésilien est encore d'actualité, car elle démontre comment le Brésil est un pays qui n'a pas su dépasser un certain nombre de choses et qui s'est sclérosé. Il note que le PCB [Parti communiste brésilien] était le parti d'organisation de masse et de la classe des travailleurs, qui a pourtant fini par faire une alliance avec les progressistes de la bourgeoisie nationale et à favoriser une transformation lente et graduelle. L'industrialisation, l'approfondissement des processus de redistribution, tout ça ce n'était que de la poudre aux yeux. Car qu'est-ce qui se passe réellement? Un coup militaire. Et le parti communiste ne s'est pas mis en tête qu'il pouvait y avoir un coup d'état. Le coup s'est produit sans réaction de la part de l'opposition. Je dirais que la dictature [de 1964] ne dura pas seulement 20 ans, mais qu'elle reste encore au pouvoir jusqu'aujourd'hui : ses éléments fondamentaux, ses structures administratives furent créés par la constitution de 1967 et n'ont jamais été modifiés. La partie de la constitution de 1988 sur la sécurité nationale est une copie de la constitution de 1967. Jusqu'aujourd'hui, la gauche n'a rien fait de nouveau, elle reste attachée au même discours et produit les mêmes résultats. Ceci démontre à quel point l'analyse de Marighella était tout à fait correcte, qu'il remarque bien à quel point la gauche est attachée à une même idée de développement. Une idée qui permet juste de redistribuer un peu plus aux pauvres, un peu comme ce que disait Lula: «Ce que je veux, c'est que le travailleur de General Motors puisse acheter sa coccinelle et descendre à Guarujá ". S'il ne s'agit que de cela, nous n'avons pas besoin de la gauche. Si 
c'est cela le grand horizon de la gauche brésilienne, il ne faut pas s'étonner de ce à quoi nous sommes en train d'assister.

Question : Doit-on alors se réjouir de la fin de la gauche?

Vladimir Safatle : Je pense qu'il n'y avait pas d'autres alternatives. C'est évident depuis 2013 qu'elle n'était pas à la hauteur des évènements. Mais quand je dis la gauche, je ne parle pas simplement du PT. Toute la gauche brésilienne a démontré les mêmes limites. L'autocritique vaut pour tout le monde. Elle a une incapacité chronique à la faire, parce qu'elle n'a rien à offrir en l'état, parce que, si elle l'a fait, elle ne sait plus où elle va.

\section{BIBLIOGRAPHIE}

ADORNO, T. Dialética Negativa. Rio de Janeiro: Jorge Zahar Ed., 2009.

FREUD, S. L'avenir d'une illusion, traduction fr. Marie Bonaparte (1882-1962, revue par l'auteur1932) PUF, Paris, 1973, Edition de l'UQAC, http://dx.doi.org/doi:10.1522/030148890 FREUD, Obras completas - 0 mal-estar na civilização, Novas conferências introdutórias à psicanálise e outros textos (1930-1936). Trad. Paulo César de Souza. São Paulo: Companhia das Letras, 2010. v. 18.

HABERMAS, J. O discurso filosófico da modernidade. São Paulo: Martins Fontes, 2002.

LACLAU, E. Razão populista. São Paulo: Ed. Três Estrelas, 2013.

LAPLANCHE, J; PONTALIS, J. B. Dicionário de Psicanálise. São Paulo: Martins Fontes, 2000.

MARCUSE, H. « Ecologie et Révolution », Le Nouvel Observateur, édition du 13 juin 1973.

MARCUSE, Sommes-nous déjà des hommes ? Textes et entretiens, « Ecologie et la critique de la société moderne ", Alboussière, QS ? Editions, 2018.

MARCUSE, Contre-révolution et révolte, chapitre II « Nature et révolution », Paris, Seuil, 1973.

MIÉVILLE, C. Outubro: história da Revolução Russa. São Paulo: Boitempo, 2017.Ecology and the Critique of Society Today.

PEREIRA, M. Pânico e desamparo: Um estudo psicanalítico. São Paulo: Escuta, 1999.

ROSA, J. G. Grande Sertão: Veredas. Rio de Janeiro: Nova Fronteira, 2006.

ROSA, No Urubuquaquá, No Pinhém. Rio de Janeiro, Nova Fronteira, 2001.

SAFATLE, V. A paixão do negativo: Lacan e dialética. São Paulo: UNESP, 2006.

SAFATLE O circuito dos afetos: corpos políticos, desamparo, fim do indivíduo. São Paulo: Cosac Naify, 2015.

SAFATLE Federn, Kelsen, Laclau e a dinâmica anti-institucional da democracia (pp. 51-62). In.: HOFFMANN, C.; BIRMAN, J. (org.). Psicanálise e Política: uma nova leitura do populismo. São Paulo: Insituto Lagange/Université Paris Diderot, 2018. 
SAFATLE Dar corpo ao impossível: o sentido da dialética a partir de Theodor Adorno. Belo Horizonte: Autêntica Editora, 2019.

\section{NOTES}

1. Hilflosigkeit est le mot que Freud emploie au long de son œuvre dans différents contextes : 1) celui de la génétique ; 2) de la totale dépendance du bébé avec sa mère (ce qui «implique son omnipotence totale», qui «influence ainsi de façon décisive la structuration psychique »);3) (celui dont il est question ici) dans le cadre d'une théorie de l'angoisse, là où « l'état de la détresse devient le prototype de la situation traumatique ». C'est ainsi que, dans Inhibition, symptôme et angoisse (Hemmung, Symptom und Angst, 1926), Freud reconnaît une caractéristique commune aux «dangers internes»: la perte, ou la séparation, qui provoque une augmentation progressive de la tension, jusqu'au point où, dans un cas extrême, le sujet se voit incapable de dominer les excitations, étant submergé par elles " (LAPLANCHE et PONTALIS, 2000, p. 76). D'après Pereira (1999), Freud, dans ses écrits plus tardifs, abandonnant un peu la lecture génétique et organiciste, finira par décrire l'hilflosigkeit, notamment à partir de son livre Le Futur est une Illusion, comme « la condition ultime du manque des garanties du fonctionnement psychique, que l'homme tente d'affronter quand il se libère de toutes les illusions protectrices qu'il crée pour lui-même » (PEREIRA, 1999).

2. V. Safatle, O circuito dos afetos: corpos políticos, desamparo, fim do indivíduo, Cosac Naify, São Paulo, 2015, p. 220.

3. Propos de Max Horkeimer recueillis par Hellmut Karasek en 1969 pour la télévision allemande dans le documentaire Porträt eines Aufklärers. Disponible à l'adresse suivante: https:// www.youtube.com/watch?v=npDU_Plntc4\&t=0s.

4. V. Safatle, Dar corpo ao impossível: o sentido da dialética a partir de Theodor Adorno, Autêntica Editora, Belo Horizonte, 2019.

5. V. Safatle, A paixão do negativo: Lacan e dialética, UNESP, São Paulo, 2006.

6. Freud, L'avenir d'une illusion, traduction fr. Marie Bonaparte (1882-1962, revue par l'auteur1932) PUF, Paris, 1973, Edition de l'UQAC, p. 43, http://dx.doi.org/doi:10.1522/030148890.

7. «Paix, pain et terre » furent les mots d'ordre, tenus pour avoir été élaborés par Lénine dans ses célèbres Thèses d'Avril, qui ont fini par devenir le grand slogan unificateur des masses populaires tout au long du processus révolutionnaire en Russie, entre mars et novembre 1917. Dans ce texte (puis dans la conscience de ses continuateurs), chacun de ces mots représente une revendication politique précise : la « paix » est en faveur de la sortie de l'empire russe de Nicolas II de la Première Guerre Mondiale ; la « terre » désigne une réforme agraire immédiate, dans une société dominée par une aristocratie rurales et dont les rapports économiques et de travail étaient jusqu'alors inscrits dans des relations de servitude ; enfin le " paix " réclame de la nourriture pour tous, dans une Russie dévastée par la famine, due aux efforts de guerre et à l'expansion impérialiste de la part du régime tzariste (voir le premier chapitre de l'ouvrage non traduit de China Miéville, Outubro. História da Revolução Russa, Boitempo, São Paulo, 2017).

8. Economiste orthodoxe brésilien, issu de l'Ecole de Chicago, nommé ministre des Finances par Dilma Rousseff en 2014, après avoir été choisi par l'ancien président Lula Ignacio da Silva, de 2003 à 2006, comme secrétaire du Trésor national. Connu pour avoir mis en place une série de mesures d'austérité et de coupes budgétaires, mises en échec par l'entrée en récession du Brésil en fin 2015, il est remplacé par Nelson Barbosa en 2016 [Nldr].

9. Surnommée "Miss Motosserra" ("Miss Tronçonneuse") Katia Abreu fait partie de l'élite agraire brésilienne et a été présidente de la CNA, un important syndicat et lobby agricole. Elle a été nommée ministre de l'Agriculture par Dilma Rousseff de 2015 à 2016 [Nldr]. 
10. Durant l'été 2017, le directeur du journal libéral The Prospect, a reçu un appel de Steve Bannon. Au cours de cette discussion, il déclare à propos du parti des Démocrates : «the longer they talk about identity politics, I got 'em. I want them to talk about racism every day. If the left is focused on race and identity, and we go with economic nationalism, we can crush the Democrats. » Source : Robert Kuttner, «Steve Bannon, Unrepentant », The Prospect, 16/09/2017, https://prospect.org/power/steve-bannon-unrepentant/.

11. In « Ecologie et Révolution », Le Nouvel Observateur, édition du 13 juin 1973.

12. In Sommes-nous déjà des hommes? Textes et entretiens, «Ecologie et la critique de la société moderne ", Alboussière, QS ? Editions, 2018.

13. In Contre-révolution et révolte, chapitre II « Nature et révolution », Paris, Seuil, 1973.

14. Mouvement artistique né au début des années 20 au Mexique. Il prône une ouverture de l'œuvre d'art à tout le peuple, prolétaires et autochtones. Les peintures muralistes représentent l'exaltation révolutionnaire (L'Arsenal, Diego Rivera), les souffrances du prolétariat (Trinité Révolutionnaire, José Clémente Orozco), l'âme indienne aspiré par la colonisation (Le Franciscain, Orozco).

15. Architecte, il est à l'origine du plan d'urbanisme de la nouvelle capitale brésilienne, Brasilia.

16. Mot brésilien spécifique qui désigne la zone semi-aride du Nordeste du Brésil.

17. J. G. Rosa, No Urubuquaquá, No Pinhém, Nova Fronteira, Rio de Janeiro, 2001, p. 36.

18. Député et président du Parlement national de 2005 à 2007, membre du Parti socialiste brésilien.

19. En 2010, le président Lula donne son accord à la construction d'un barrage dans la région de Belo Monte, en Amazonie, territoire habité par des populations indigènes. Source : «Lula donne son accord à la construction d'un barrage très controversé sur l'Amazone", Le Monde, 27/08/2010, https://www.lemonde.fr/ameriques/article/2010/08/27/lula-donne-son-accord-ala-construction-d-un-barrage-tres-controverse-sur-l-amazone_1403322_3222.html.

20. Références aux ruptures de barrages miniers de Mariana (novembre 2015) et de Brumadinho (janvier 2019), contrôlés par les grandes d'exploitation minière Vale do Rio Doce et Samarco.

21. Ancien maire de São Paulo de 2012 à 2016, candidat à la présidentielle de 2018 face à Bolsonaro, il remplace Lula à la suite de la condamnation l'empêchant de se présenter aux élections.

22. Député fédéral du Ceará, membre du parti de centre gauche PDT, Parti Démocratique Travailliste.

\section{NOTES DE FIN}

a.

\section{AUTEURS}

\section{VLADIMIR SAFATLE}

Professeur de philosophie à l'Université de Sao Paolo, Brésil 